\title{
Farmacoeconomía. Eficiencia y uso racional de los medicamentos
}

\author{
Manuel M. Collazo Herrera*
}

Centro de Investigación y Desarrollo de Medicamentos (CIDEM), Ciudad de La Habana, Cuba

*Correspondencia:

Centro de Investigación y Desarrollo de Medicamentos (CIDEM)

Calle 26 No. 1605 entre Ave. Rancho

Boyeros y Calzada de Puentes Grandes Nuevo Vedado, Plaza de la Revolución C.P. 10600

Ciudad de La Habana, Cuba.

E-mail: cidem@infomed.sld.cu
Este artículo expone como la farmacoeconomía contribuye a incrementar los niveles de eficiencia en la esfera de la utilización de los medicamentos. Mediante los lineamientos de trabajo que persigue esta disciplina, se podrán llevar a vías de realización diferentes aplicaciones en el campo de la prestación de los servicios sanitarios, y en el proceso de obtención de nuevos fármacos por parte de la industria farmacéutica. Como resultado del enfoque actual que le dan las autoridades sanitarias a la farmacoeconomía, la industria farmacéutica a escala internacional se ha empezado a interesar por dichas técnicas analíticas y a utilizarlas para desarrollar sus estrategias de investigación-desarrollo, producción y comercialización de sus productos; lo que constituye uno de los esfuerzos iniciales más importantes realizados, encaminados a lograr que la evaluación económica comience a incorporarse al proceso integral de la circulación de los medicamentos. Como conclusión de este análisis, se puede afirmar que la evaluación farmacoeconómica contribuye a darle un uso más efectivo y eficiente de los medicamentos que se emplean en los tratamientos para combatir las distintas enfermedades. En el contexto nacional del sistema sanitario, la farmacoeconomía permite articular de forma cohesionada, los intereses de la industria farmacéutica con los del sector de la prestación de los servicios de salud, demostrando así la aplicación de esta útil herramienta de trabajo en la toma de decisiones sanitarias; y poder elaborar de esta manera, las políticas y estrategias para el uso racional de los medicamentos, sobre la base de su efectividad clínica comprobada y los costos de la farmacoterapia empleada.
Unitermos

- Farmacoeconomía

- Medicamentos/uso racional

- Costos del tratamiento

- Política sanitaria

\section{INTRODUCCION}

La aplicación de los conocimientos y técnicas de la evaluación económica al campo de los medicamentos permite centrar el análisis en el tratamiento de los problemas que plantea una adecuada prescripción, tanto desde el punto de vista de la efectividad en términos de salud, como de la eficiencia; es decir, de la obtención de un beneficio social a un costo razonable. La farmacoeconomía es, por lo tanto, una herramienta útil para medir los efectos que tienen las 
decisiones sobre los recursos disponibles y también a las posibilidades existentes para cuantificar el impacto, en términos de salud, de una intervención sanitaria.

Como consecuencia del enfoque actual que le dan las autoridades sanitarias a la farmacoeconomía, la industria farmacéutica a nivel mundial se ha empezado a interesar por dichas técnicas analíticas y a utilizarlas para desarrollar sus estrategias de investigación-desarrollo, producción y comercialización de sus productos.

La evaluación económica de los medicamentos no es una panacea frente a los problemas a los que se enfrentan diariamente los profesionales de la salud, pero permite reducir las arbitrariedades en la toma de decisiones sobre el uso de los recursos disponibles, de manera que constituye un instrumento indispensable para mejorar la práctica sanitaria y para conseguir mejores resultados a un costo asumible para la economía de un país.

El objetivo del presente trabajo, es realizar una investigación bibliográfica que brinde una panorámica actual de la evaluación económica aplicada al campo de la farmacoterapia. La aplicación práctica de estas técnicas de análisis al sistema sanitario representa uno de los esfuerzos iniciales más importantes realizados en algunos países, encaminados a lograr que la evaluación farmacoeconómica comience a incorporarse al proceso de utilización racional de los medicamentos, demostrando así su aplicación en la toma de decisiones sanitarias sobre la base de la efectividad terapéutica y los costos del tratamiento.

\section{INCORPORACIÓN DEL ANÁLISIS ECONÓMICO AL CAMPO DE LOS FÁRMACOS}

Los medicamentos han constituido siempre un componente importante de la vida del hombre y de las organizaciones sociales. Su papel es esencial para prevenir enfermedades, proteger y preservar la salud (Vernengo, 1996).

En este sentido, se puede definir al medicamento como una preparación o formulación farmacéutica empleada para la prevención, diagnóstico y/o tratamiento de una enfermedad o estado patológico; o para modificar sistemas fisiológicos en beneficio de la persona a quién se le administra (Vernengo, 1993). Aunque los medicamentos no son suficientes por sí solos para proporcionar una atención adecuada, juegan un papel importante en la protección, mantenimiento y restauración de la salud.

El medicamento no solo es un componente esencial de la atención de salud, un símbolo del tratamiento sino que, además, es una mercancía y un bien de transacción, producto de la industria moderna; también, es un objeto de comercio y de venta. Como insumo para las acciones de salud, representan un costo que tiene repercusiones importantes en los presupuestos oficiales y en el de las personas (Vernengo, 1996).

Desde el punto de vista económico, los medicamentos son bienes de consumo que son aquellos productos elaborados para ser utilizados, tanto por el consumo productivo como por el consumo personal. Por lo tanto, se puede definir al medicamento como un producto, como cualquier bien material que posea valor para el consumidor o usuario, y sea susceptible de satisfacer una demanda y reporte un ingreso monetario al productor.

Los medicamentos son insumos importantes en los servicios de salud y objeto de transacciones comerciales. Las personas los obtienen en las farmacias comunitarias $\mathrm{y}$, para su adquisición, emplean una proporción elevada de sus ingresos. Como la relación que guarda el medicamento como objeto de comercialización y su valor de uso es tan decisivo, que originó el concepto del medicamento como "bien social" (Vernengo, 1996).

Las modalidades y razones del consumo de medicamentos están condicionadas por diversos factores interactuantes, entre los que cabe mencionar: las prácticas terapéuticas y de prescripción médica, la actitud de los pacientes en su relación con los médicos, la percepción de la población en relación a las propiedades de los medicamentos, los recursos económicos de las personas, la disponibilidad de fondos públicos y privados para su adquisición y distribución, y las actividades de promoción de la industria farmacéutica. El empleo está determinado también por aspectos culturales y por otras características específicas de la población consumidora como, por ejemplo, las enfermedades prevalentes, y los recursos tecnológicos y económicos disponibles.

Por otra parte, en nuestros días, la atención de la salud es objeto de una intensa actividad comercial que induce al consumo excesivo de sus servicios y, en particular, al empleo de todos los recursos tecnológicos. Esas condiciones refuerzan el papel de los medicamentos.

El carácter lucrativo de la actividad industrial de producción y de comercialización de los medicamentos ha propiciado desvíos importantes como la producción excesiva de medicamentos innecesarios, ineficaces o con potencial tóxico, la prescripción irracional, la automedicación, etc. Ello, a su vez, afecta las actividades de enseñanza, información y consumo (Lobo, Velázquez, 1997).

El gasto en medicamentos no cesa de aumentar en todo el mundo, con crecientes desigualdades entre los países. El mercado mundial ya supera la cifra de US \$ 250,000 millones anuales, de los cuales, cerca de $7 \%$ corresponde a América Latina. En términos globales, la tercera parte de la población mundial consume el $66 \%$ de la 
producción farmacéutica, mientras el resto de la población consume solo el 34\% de este total (Vernengo, 1996).

El medicamento se ha constituido en un elemento complejo cuyo uso implica considerar no sólo los aspectos farmacoterapéuticos, sanitarios y sociales sino, también, los de carácter económico, industrial, financiero y comercial. Así, existe la necesidad de concebir una política que ligue armoniosamente a la industria y el comercio, por un lado, y al consumidor, el médico y la autoridad sanitaria por el otro. La mayor parte de los países enfrenta una escasez de recursos en el área de la salud, que les obliga a la contención, reorientación y racionalización de sus gastos (Lobo, Velázquez, 1997)

Esta situación y la ausencia de una política y de programas definidos conducen a graves problemas de financiamiento del sistema de atención de la salud con el consecuente aumento del gasto privado y la disminución de los recursos destinados a la asistencia farmacéutica. Esto es aún más graves en los países en vías de desarrollo en donde, además, se produce el desabastecimiento de los hospitales y centros de salud que atienden a los sectores más necesitados de la población. Los servicios públicos se caracterizan por una insuficiente disponibilidad de medicamentos, material médico-quirúrgico y otros insumos que influye negativamente sobre la calidad de la atención y la utilización de los servicios (Madrid, Velázquez, Fefer, 1998).

Los problemas de salud constituyen un asunto político de primer orden, tanto en los países industriales como en los países en vías de desarrollo. El debate político se desarrolla actualmente en torno al aumento incesante de los costos, las dificultades de la financiación pública y la ausencia de cobertura real para toda la población. Es evidente, entonces, que los países deben estructurar una política de salud orientada hacia el empleo más racional de los recursos y la ampliación y funcionamiento adecuado de los servicios de salud. Esa política debe generar estrategias para administrar los recursos-siempre escasos-humanos, técnicos, económicos y financieros para obtener los beneficios más amplios posibles.

\section{NECESIDAD DEL USO RACIONAL DE LOS MEDICAMENTOS}

Las políticas de medicamentos deben encaminarse, primordialmente, a racionalizar y estimular su uso apropiado, ajustado a los objetivos globales y particulares de una política de salud que asegure la accesibilidad a las prestaciones de salud y a los medicamentos. Deben procurarse, adicionalmente, estrategias para la contención de los gastos en medicamentos o su reducción en el marco de accio- nes que tiendan a controlar el costo de las prestaciones de salud. Se trata, entonces, de establecer un programa concebido para atender las necesidades con el objetivo fundamental de satisfacerlas mediante productos de eficacia e inocuidad demostradas, de la mejor calidad posible, y a precios y costos razonables o los más bajos posibles (Madrid, Velázquez, Fefer, 1998).

Para un uso racional es preciso que se prescriba el medicamento apropiado, que se disponga de este medicamento oportunamente y a un precio asequible, que se despache en las condiciones debidas y que se tome en las dosis indicadas y a los intervalos, y durante el tiempo prescrito. El medicamento apropiado ha de ser eficaz y de calidad y seguridad aceptadas (Arias, 1999). Por lo tanto, se deberá concebir el uso racional de los medicamentos como un conjunto de medidas cuyo objetivo es asegurar el acceso de la población a los medicamentos que se necesitan para una asistencia sanitaria efectiva, a un costo asumible para la sociedad (SOIKOS, 1996).

El suministro de medicamentos, desde el punto de vista administrativo, tiene como finalidad garantizar a toda la población la disponibilidad, asequibilidad y uso racional de medicamentos en condiciones óptimas de calidad, seguridad y eficacia. Se concibe como un sistema constituido por etapas secuenciales interrelacionadas intra e interdependientes e integradas, que demandan una cuidadosa coordinación y riguroso controles de calidad y eficiencia. Ciertamente, sólo se puede practicar una medicina efectiva donde haya una administración eficiente de medicamentos (OMS, 1990).

El establecimiento de modelos, guías de tratamiento $y$, específicamente, de tratamientos estandarizados como parte de los programas que instituyen formularios de medicamentos esenciales - tiene por objeto establecer mayor racionalidad en la utilización de los medicamentos $\mathrm{y}$, subsidiariamente, un sistema para la contención de gastos en la atención farmacoterapéutica (OMS, 1989).

\section{IMPORTANCIA DE LA EVALUACIÓN ECONÓMICO-SOCIAL DE LOS MEDICAMENTOS}

Toda esta situación ha dado como resultado que, en la actualidad, se le dé importancia a la evaluación del impacto social y económico de los medicamentos sin dejar de considerar los aspectos técnicos y científicos relacionados con su eficacia, inocuidad y calidad. En ese contexto, se han generado nuevas propuestas para el desarrollo del área farmacéutica englobadas en el concepto de evaluación socioeconómica de los medicamentos. En los últimos años, los gobiernos han propiciado una serie de medidas que pro- 
curan la contención de gastos en salud y en medicamentos frente al aumento porcentual sostenido de estos gastos para toda la sociedad y para las personas individuales.

Entre los diversos aspectos que deben considerarse en este contexto antes de autorizar su introducción al mercado, se pueden mencionar los siguientes: potencialidad para inducir la automedicación, indicaciones terapéuticas con relación a los hábitos de prescripción, posibilidades generadas por las formas de presentación del medicamento en términos del cumplimiento o incumplimiento de los tratamientos y la influencia que el sistema de atención tendrá en su utilización, así como el costo del tratamiento con relación a otros (Haaijer, Ruskamp \& MNG, 1993). Evaluaciones de esta naturaleza tendrían importancia en los sistemas de atención de la salud para la utilización de formularios más reducidos.

Con relación a los aspectos económicos y financieros de la utilización de los medicamentos, la evaluación deberá tener como base una consideración relativa de costo, beneficio y riesgos. La incorporación de nuevos medicamentos al sistema de salud y al mercado de un país debería tomar en cuenta estos aspectos financieros y económicos de la utilización de los medicamentos (Haaijer, Ruskamp, MNG, 1993; Manley, 1996).

Para evaluar el empleo de los medicamentos, desde un punto de vista más global y no solamente en términos farmacoterapéuticos, es preciso realizar evaluaciones económicas de los medicamentos. Los análisis económicos de este tipo, referidos a medicamentos específicos o a clases o tipos farmacológicos, deberían publicarse como cualquier otro resultado de investigación y ser accesibles públicamente o, quizás, sería necesario que esa información se proveerá conjuntamente con la referida a la eficacia e inocuidad en relación a las indicaciones, dosis a utilizar, tiempo de tratamiento, etc. En realidad, se trata de la comparación entre productos farmacéuticos y otros métodos de tratamiento con respecto a sus propiedades terapéuticas y a los costos de su utilización como métodos alternativos, y la factibilidad de aplicar conceptos económicos de costo/beneficio para la toma de decisiones en el sector farmacéutico (Collazo et al., 2002).

En países como Australia y Canadá, se agregan a estos estudios acciones administrativas en las que las autoridades provinciales incorporan medicamentos a sus formularios terapéuticos basándose sobre la presentación de costos relativos, relacionados o no a terapéuticas farmacológicas alternativas (CCOHT, 1994).

La industria farmacéutica estabeleció en su estructura interna, grupos u organizaciones que puedan preparar estudios destinados a evaluar positivamente sus productos (McGhan, Lemus, 1992). Situaciones de este tipo estarían facilitando la revalorización de los medicamentos antiguos de uso prolongado y reconocidos dentro de la terapéutica médica (que fueron o pudieron haber sido reemplazados por medicamentos más recientes).

Estos tipos de estudios farmacoeconómicos han reintroducidos mecanismos de evaluación relativa de medicamentos, mediante los cuales se comparan productos farmacéuticos diferentes y se valora la sustitución terapéutica entre productos terapéuticamente similares (aun cuando estén constituidos por diferentes principios activos o diferentes formas farmacéuticas y con los mismos principios activos) (Drummond et al., 1993).

En Canadá y Australia, se toman en cuenta todas estas variables sociales, económicas, médicas y sanitarias para evaluar la incorporación de un medicamento dentro del sistema de beneficios farmacéuticos y de los formularios terapéuticos utilizados en sus sistemas de atención de la salud (Manley, 1996).

\section{EL CRITERIO FARMACOECONÓMICO PARA EVALUAR LA EFICIENCIA DEL TRATAMIENTO}

La evaluación económica de medicamentos cuyo sinónimo es farmacoeconomía puede expresarse como la aplicación del análisis económico al campo de los medicamentos (Velázquez, 1999); y también, se puede referir más específicamente a la aplicación de la teoría económica a la farmacoterapia (Milne, 1992). Puesto que toda evaluación se basa en la comparación, la evaluación económica de medicamentos podría definirse como la determinación de la eficiencia (relación entre costos y efectos) de un tratamiento farmacológico y su comparación con la de otras opciones, con el fin de seleccionar aquella con una relación costo/efecto más favorable (Sacristán del Casti1lo, 1995).

De esta manera, la evaluación económica de medicamentos puede contribuir a armonizar las necesidades crecientes que la población demanda en materia de salud con los recursos económicos disponibles (Eisemberg, Glick, Koffer, 1989). La selección de una determinada opción terapéutica según su eficiencia; es decir, considerando simultáneamente sus costos y sus beneficios, supone un avance considerable respecto a otro tipo de criterios más parciales (Luce, Elixhauser, 1990).

El criterio de eficiencia es aplicable por diferentes agentes en distintos ámbitos del sector sanitario. Las recientes experiencias internacionales muestran que las técnicas de evaluación económicas de medicamentos están cobrando cada vez una mayor importancia en la toma de decisiones sanitarias (Drummond, Stoddart, Torrance, 1990), proporcionando una inestimable ayuda para lograr 
que estas estén basadas en criterios más racionales, objetivos y transparentes.

La evaluación económica de medicamentos utiliza los métodos del análisis económico, lo cual significa que todos los profesionales que trabajan en este campo e interpretan información proveniente de éste necesitan unos conocimientos básicos sobre los términos y conceptos que son actualmente el denominador común tanto de la evaluación económica como de la economía de la salud. Es por ello, que la farmacoeconomía es una actividad que requiere equipos multidisciplinarios y, de hecho, una elevada proporción de los analistas que llevan a cabo estudios en este campo no son economistas sino que proviene del campo de las ciencias de la salud, de la estadística, de la investigación operativa, etc, y a menudo no han tenido un aprendizaje formal en disciplinas económicas.

De esta forma, el conocimiento de los conceptos farmacoeconómicos por parte de los profesionales que laboran tanto en el sistema sanitario como en la industria farmacéutica, posibilitan la introducción de una cultura económica en sus tareas habituales; es decir, a considerar la relación existente entre los costos y los resultados de la farmacoterapia empleada.

Desde una perspectiva clínica, la utilización de una tecnología se justifica si su efectividad (la consecución de un objetivo en condiciones reales) o, por lo menos, su eficacia (la consecución de un objetivo en condiciones ideales) es positiva. Sin embargo, desde la perspectiva económica el acento se pone en la eficiencia (Badia, Rovira, 1994).

Pensar en términos de eficiencia implica considerar la efectividad de un proceso con relación a los recursos que requiere (Drummond, 1994). La razón es obvia, dado que los recursos son limitados respecto a sus posibles aplicaciones, cuanto menos recursos sean necesarios para conseguir un determinado objetivo, tanto mejor; ya que así quedarán más recursos disponibles para lograr otros propósitos en términos de salud (Bootman, Towsend, Mc Ghan, 1991).

La evaluación económica de medicamentos constituye un campo de conocimiento, y un instrumento efectivo de trabajo para lograr un óptimo aprovechamiento de los recursos humanos, materiales y financieros que se destinan para los servicios de salud (Collazo, Flores, 2000). Su aplicación práctica se basa en el principio de que el paciente debe recibir los recursos que requiere para mantener su salud, y está orientada al desarrollo de estudios que permitan seguir ese principio mediante la eficiencia y racionalidad en el uso de los fármacos (Collazo, Rovira, 1997).

La evaluación económica de tecnología y programas sanitarios y, especialmente de productos farmacéuticos, se está utilizando cada vez con mayor frecuencia para hacer frente al hecho de que los recursos deben utilizarse con la mayor eficiencia posible para lograr el máximo beneficio de los pacientes (PharmacoEconomics, 1994). Es por ello, que la industria farmacéutica se está involucrando cada vez más en la evaluación económica de los medicamentos que desarrolla, ya que las aplicaciones prácticas de los estudios farmacoeconómicos pueden ser útiles en multitud de situaciones relacionadas con la toma de decisiones (CCOHT, 1994).

En la actualidad, y dado el elevado costo que supone el desarrollo de un nuevo producto; existe dentro de las compañías farmacéuticas una clara vocación y orientación en proporcionar fármacos cada vez más eficientes, a la vez más eficaces, mejor tolerados y de mayor calidad; lo que supondrá un claro avance y mejora dentro del arsenal terapéutico disponible (Soto, 1999). Por este motivo dentro de la industria farmacéutica, cada vez se están diseñando y realizando análisis de evaluación económica de medicamentos con más asiduidad durante todo el ciclo de vida; tanto durante los primeros estadios de su desarrollo como una vez hayan sido comercializado (Anderson, 1995). Este hecho refleja la necesidad de prioridad de inversión de los recursos existentes, y escoger los medicamentos que presenten una mejor relación costo-efectividad; y que por lo tanto, logren el mejor beneficio terapéutico con el menor costo asociado posible (Sacristán et al., 1994).

En sociedades industrializadas capaces de regular y controlar los costos de producción de bienes de salud y donde la cobertura sanitaria es prácticamente total, la farmacoeconomía podrá sin duda ayudar a mejorar la toma de decisiones clínicas, sobre todo cuando se debe escoger entre varios tratamientos similares. Todo ejercicio, sin embargo, debe estar enfocado en la perspectiva no solo de controlar costos, sino de mejorar la eficiencia (Evans, 1992) para alcanzar la equidad en el acceso a los servicios de salud.

En los países en desarrollo, donde una parte importante de la población no tiene acceso a medicamentos y donde muchas personas mueren a causa de enfermedades para las cuales existen medicamentos preventivos, la farmacoeconomía tendrá un enfoque y unas características muy diferentes. Más que contención de costos, se buscará racionalizar los recursos existentes y conseguir fuentes suplementarias de financiamiento, de tal forma que garantice la extensión de la cobertura a toda la población (Velázquez, 1999).

Debido a esta falta de medicamentos para tratar las enfermedades comunes en los países en desarrollo, como la tuberculosis y la malaria, y el alto costo de los medicamentos contra las enfermedades de distribución mundial, como el SIDA, es que se están empezando a considerar a 
estos fármacos como "bienes públicos mundiales". En consecuencia, la comunidad internacional está creando un Fondo Global de Salud para facilitar tanto el desarrollo de nuevos medicamentos contra esas enfermedades como el acceso a ellos (Vieira, 2002).

Un mundo sin poliomielitis representa un ahorro anual en costos de vacunación de US \$ 1500 000. Una sola inversión de US \$ 370 millones redundará en ahorros de US $\$ 1,5$ mil millones anuales por tanto tiempo como sea imaginable. Hoy en día el producto nacional bruto (PNB) de África sería probablemente de unos US \$ 100000 millones más si la malaria hubiera sido controlada hace 30 años, cuando se aplicaron las primeras medidas eficaces de control (Collazo et al., 2002).

Mejorar el acceso a medicamentos para las personas viviendo con VIH/SIDA supone un reto para todos los países, y mucho mayor aún para los países en vías de desarrollo. De los más de 40 millones de personas viviendo con VIH/SIDA en todo el mundo, más del $95 \%$ vive en países en vías de desarrollo. Muchos de ellos no tienen acceso ni tan siquiera a los medicamentos más básicos para el tratamiento de infecciones relacionadas con el VIH ni para otras enfermedades (OMS, 2002)

El elevado precio de muchos de los medicamentos contra el VIH, especialmente los antirretrovirales, suministrados por proveedores comunes es uno de los principales obstáculos para su disponibilidad en los países en vías de desarrollo (OMS, 2002).

En este sentido, el debate sobre el acuerdo de protección de la propiedad intelectual (TRIPS) en la reciente Conferencia Ministerial de la Organización Mundial del Comercio (OMC) en Doha, Qatar, reconoció la importancia de ejecutar e interpretar el TRIPS "de una manera que apoye el derecho de los miembros de $\mathrm{OMC}$ a proteger la salud pública, y en particular, promover el acceso a los medicamentos de toda la población" (OMS, 2001). Como consecuencia, políticas comerciales derivadas del TRIPS, como la fijación negociada de precios escalonados, la concesión obligatoria de licencias en el caso de emergencias sanitarias, y la transferencia de tecnologías a países con menor capacidad productiva, pueden ayudar a reducir las brechas en el acceso a medicamentos que hoy se observa entre los países desarrollados y en vías de desarrollo (Vieira, 2002).

Dada esta situación existente en el mundo subdesarrollado, una farmacoeconomía de este tipo estaría más centrada en la selección de fármacos y la evaluación económica debería agregarse a los criterios terapéuticos para la elaboración de formularios, listas básicas o sistemas de reembolso. La evaluación económica de medicamento sería también un elemento más para promover lo que la
Organización Mundial de la Salud (OMS) ha llamado, el uso racional del medicamento (Velázquez, 1999).

También puede darse el caso de países del Tercer Mundo que cuenta con un sistema sanitario comparable a los que tienen los países desarrollados, como por ejemplo, el Sistema de Salud Pública que tiene la República de Cuba. En esta situación, la farmacoeconomía tendrá un doble propósito: racionalizar el uso de los medicamentos seleccionados y mejorar los niveles de eficiencia de los tratamientos (Collazo, Casademunt, 2001).

La incorporación de estas técnicas de análisis por parte de la industria farmacéutica y el sistema de salud, representa una vía muy importante para lograr que la evaluación económica comience a incorporarse en toda la esfera de la circulación de los medicamentos; que comprende las etapas siguientes: selección, adquisición, distribución y prescripción de fármacos.

El objetivo de estos estudios farmacoeconómicos es elaborar y poner en práctica en el ámbito nacional, una política para el uso racional de los medicamentos que esté acorde a la situación económico-financiera que tiene el país; así como de elevar los niveles de eficiencia de los tratamientos farmacológicos para el sistema sanitario. De esta forma, se posibilitan conjugar los intereses de la industria farmacéutica con el área de la prestación de servicios de salud; dada la coordinación de trabajo y vínculos existentes entre ambos sectores, que les permitan trazar las proyecciones estratégicas conjuntas de trabajo en este sentido, para obtener un mejor uso de los fármacos sobre la base de su efectividad terapéutica y la conveniencia económica de su utilización.

\section{CONCLUSIONES}

1. Para lograr un uso más eficiente de los recursos disponibles, es para lo que se lleva a cabo cada vez más los estudios farmacoeconómicos, que permiten comparar las diferentes alternativas u opciones de tratamientos, y poder evaluar el valor de los beneficios, la efectividad; y el efecto sobre la cantidad y calidad de vida de los pacientes.

2. La colaboración de profesionales de distintas formación es básica para el completo desarrollo y difusión de las técnicas para los análisis farmacoeconómicos. Debe promoverse que el personal que labora con los medicamentos, se vayan habituando a realizar e interpretar estos estudios para contribuir a la mejor utilización de los recursos sanitarios disponibles.

3. La evaluación económica de medicamentos es una temática de interés para el campo sanitario y pese a las limitaciones que pudiera tener, se encuentra disponible 
como herramienta útil de trabajo para orientar la toma de decisiones tanto en el ámbito de la administración sanitaria, como en los servicios de salud y en las empresas farmacéuticas.

4. La implementación del criterio farmacoeconómico en los tratamientos es una de las principales estrategias de racionalización farmacoterapéutica para el Sistema Nacional de Salud, ya que permite evaluar de forma integral diferentes variables como son: la efectividad, los costos y la eficiencia de las distintas intervenciones sanitarias analizadas.

5. La farmacoeconomía posibilita conjugar los intereses de la industria farmacéutica con el sector de la prestación de servicios sanitarios, y poder elaborar de esta manera, las políticas y estrategias conjuntas de trabajo para el uso racional de los medicamentos, sobre la base del incremento de la eficiencia en los tratamientos farmacológicos.

\section{ABSTRACT \\ Pharmacoeconomics. The efficiency and rational use of pharmaceutical products}

This paper is a discussion about pharmacoeconomics contributes to increase the efficiency levels in the field of drug use. By this discipline's work guidelines, different pharmacoeconomic applications will be able to be performed, both in the field of sanitary-services offering and in the process of obtaining new drugs by the pharmaceutical industry. As a result of the sanitary authorities' present focusing on pharmacodinamics, the international pharmaceutical industry has started to become interested in such analytical techniques, and to use them in its research-development-production and marketing strategies for its products; this is one of the most important initial efforts, which are targeted to attain that economic evaluation starts to be involved in the comprehensive process of drug circulation. As a conclusion of this analysis, it can be stated that pharmacoeconomic evaluation contributes to give a more effective and efficient use to drugs used in the treatment of different diseases. Within the national context of sanitary system, pharmacoeconomics allows to assemble, in a cohesion way, the pharmaceutical industry's interests with those of health-services offering. Thus, this useful work tool's application both in the sanitary-decisions taking and being able to work out policies and strategies for the drug rational use is presented, on the base of its proved clinical effectiveness and the costs of pharmacotherapy used.
UNITERMS: Pharmacoeconomics. Drugs/rational use. Therapy cost. Sanitary policy.

\section{REFERENCIAS BIBLIOGRAFICAS}

ANDERSON, F. Why is the pharmaceutical industry investing increasing amounnts in health economics evaluations? Int. J. Tech. Assess. Health Care, v. 11, p. 750-61, 1995.

ARIAS, T.D. Glosario de medicamentos: desarrollo, evaluación y uso. Washington D. C.: Organización Panamericana de la Salud, 1999. 333 p.

BADIA, X.; ROVIRA, J. Evaluación económica de medicamentos. Un instrumento para la toma de decisión en la práctica clínica y la política sanitaria. Barcelona: Luzàn 5, 1994. cap. 2, p. 19-28.

BOOTMAN, J.L.; TOWSEND, R.J.; Mc GHAN, W.F. Principles of pharmacoeconomics. 2. ed. Cincinnati: Harvey Books Company, 1996. cap. 1, p. 5-18.

CANADIAN COORDINATING OFFICE FOR HEALTH TECHNOLOGY. Guidelines for economic evaluation of pharmaceutics. 1. ed. Ottawa: CCOHT, 1994. 40 p.

COLLAZO, M.; CASADEMUNT, N. La farmacoeconomía en la industria farmacéutica y el sistema sanitario de Cuba. Rev. Panam. Salud Pública, v.10, n.4, p. 263-7, 2001.

COLLAZO, M.; CARDENAS, J.; GONZALEZ, R.; MIYAR, R.; GALVEZ, A. M.; COSME, J. La economía de la salud: ¿debe ser de interés para el campo sanitario? Rev. Panam. Salud Pública, v. 12, n.5, p. 359-65, 2002.

COLLAZO, M.; FLORES, N. Farmacoeconomía. La evaluación de la eficiencia en los tratamientos farmacológicos. Rev. Cubana Farmacia, v. 34, n.1, p. 6369, 2000.

COLLAZO, M.; ROVIRA, J. Criterios sobre la evaluación económica de los medicamentos antineoplásicos. Rev. Cubana Oncología, v. 13, n.1, p. 23-30, 1997.

DRUMMOND, M.F. Issues in the conduct of economic evaluations of pharmaceutical products. PharmacoEconomics, v. 6, n.5, p. 405-11,1994. 
DRUMMOND, M.; RUTTEN, F.; BRENNA, A.; PINTO, C.G.; HORISBERGER, B.; JONSON B. Economic evaluation of pharmaceuticals. A European perspective. PharmacoEconomics, v. 4, n.2 p.173-86, 1993.

DRUMMOND, M.; STODDART, G.; TORRANCE, G. Métodos para la evaluación económica de los programas de atención de la salud. Madrid: Editorial Díaz de Santos,1990. cap. 3, p.23-47.

EINSEMBERG, J.M.; GLICK, H.; KOFFER, H. Pharmacoeconomics: Economic evaluation of pharmaceuticals. In: STROM B, ed. Pharmacoepidemiology. New York: Churchill Livingston, 1989. p. 77-89.

EVANS, D. The changing healthcare environment. PharmacoEconomics, v. 1, n.1, p. 5-7, 1992.

LOBO, F.; VELAZQUEZ, G. Los medicamentos ante las nuevas realidades económicas. Madrid: Colección Economía. Biblioteca Civitas Economía y Empresa. cap. 2, 1997. $270 \mathrm{p}$

LUCE, B.R.; ELIXHAUSER, A. Standards for the socioeconomics evaluation of health care services. Berlin: Springer-Verlag, 1990. cap. 3, p. 30-43.

MADRID, I.; VELAZQUEZ, G.; FEFER, E. Pharmaceutical and health sector reform in the Americas: an economic perspective. Washington D. C.: Pan American Health Organization, 1998. cap. 1, p. 1-12.

MANLEY, J. Cost-effectiveness: the need to know. Australian Prescriber, v.19, n.3 p. 58-9. 1996.

Mc GHAN, W.F., LEMUS, J.W. Guidelines for pharmacoeconomic studies. Clin. Ther., v.14, n.1, p. 48694, 1992.

MILNE, R. Editorial. PharmacoEconomics, v.1, n.1, p. 1, 1992.

PHARMACOECONOMICS. Theory into practice. Procedings of the International Conference held in Geneva, Switzerland. PharmacoEconomics, v. 6, n.4, p. 275-404, 1994.
SACRISTAN DEL CASTILLO, J.A. Farmacoeconomía y evaluación de medicamentos. Introducción. In: SACRISTAN DEL CASTILLO, J.A.; BADIALLACH, X.; ROVIRA FORNS, J. eds. Farmacoeconomía: evaluación económica de medicamentos. Madrid: Editores Médicos, 1995. cap. 1, p. 19-30.

SACRISTAN, J.A.; SOTO, J.; REVIRIEGO, J.; GALENDE, I. Farmacoeconomía: el cálculo de la eficiencia. Med. Clin., Barcelona, v. 103, p.143-149, 1994.

SOIKOS. Glosario de términos y conceptos de uso frecuente en la evaluación económica de medicamentos y programas sanitarios. Barcelona: Química Farmacéutica Bayer, 1996. 100 p.

SOTO, J. Evaluación económica de medicamentos: perspectiva de la industria farmacéutica en nuestro país. Rev. Atenc. Farm., v. 1, n.6, p. 600-8, 1999.

VELAZQUEZ, G. Farmacoeconomía: ¿evaluación científica o estrategia comercial? Rev. Panam. Salud Pública, v. 5, n. 1, p. 54-57, 1999.

VERNENGO, M.J. Elementos técnicos de una política de medicamentos genéricos. Washington D. C.: Organización Panamericana de la Salud, 1993. 47 p.

VERNENGO, M.J. Control oficial de medicamentos. Washington D. C.: Organización Panamericana de la Salud, 1996. cap. 2, p. 13-53.

VIEIRA, C. Globalización, comercio internacional y equidad en materia de salud. Rev. Panam. Salud Pública, v. 11, n.5/6, p. 425-29, 2002.

WORLD HEALTH ORGANIZATION. Cómo estimar las necesidades de medicamentos. Manual Práctico. Programa de Acción sobre Medicamentos y Vacunas Esenciales. Ginebra: WHO/DAP/88-2. Introducción, 1989. p. 1.1-1.7

WORLD HEALTH ORGANIZATION. The role of the pharmacist in the health care system. Geneva: WHO/ PHARM/DAP/90.1, 1990. cap. 3, p. 3.1-3.12. 
WORLD HEALTH ORGANIZATION. Fuente y precios de medicamentos y diagnósticos seleccionados para personas viviendo con VIH/SIDA. Proyecto conjunto de UNICEF- Secretariado ONUSIDA-OMS-MSF. Ginebra:WHO/EDM/PAR/2002.2, 2002. cap. 1, p. 1-4.
WORLD HEALTH ORGANIZATION. Implications of the Doha Declaration on the TRIPS Agreement and Public Health. Geneva: WHO/EDM/PAR/2002.3, 2001. cap.2, p. 5-13.

Recebido para publicação em 23 de junho de 2003. Aceito para publicação em 18 de outubro de 2004. 